\title{
Genetic characterization and deployment of a major gene for grain yield on chromosome arm 1BS in winter wheat
}

\author{
Chia-Cheng Kan • Haiyan Jia • Carol Powers • \\ Brett F. Carver $\cdot$ Liuling Yan (D)
}

Received: 29 September 2019 / Accepted: 6 February 2020 / Published online: 19 February 2020

(C) The Author(s) 2020

\begin{abstract}
Winter bread wheat (Triticum aestivum L., $2 n=6 \times=42$, AABBDD) cultivars "Duster" and "Billings" have occupied significant acreages in the Southern Great Plains for their outstanding yielding ability. In this study, we discovered a major quantitative trait locus (QTL) QYld.osu-1BS for grain yield in a population of 260 doubled haploid (DH) lines derived from the cross of Duster and Billings. When the population was tested under field conditions for 2 years, QYld.osu-1BS explained $13.9 \%$ and $23.5 \%$ of the total phenotypic variation. However, no crossover was observed among 40 genotyping-by-sequencing markers covering the region from the telomere to $25.3 \mathrm{Mb}$ in the population of 260 DH lines. Furthermore, no crossover was observed in the region from the telomere to $18.4 \mathrm{Mb}$, when up to 4146 individual plants within $\mathrm{F}_{2: 4}$ lines derived from the cross of Duster and Billings were screened. The 1BL1RS translocation was not observed in the region with the abnormal recombination rate in Duster or Billings. Duster is a unique haplotype in the whole exome capture
\end{abstract}

Electronic supplementary material The online version of this article (https://doi.org/10.1007/s11032-020-1106-0) contains supplementary material, which is available to authorized users.

C.-C. Kan · H. Jia · C. Powers • B. F. Carver · L. Yan $(\bowtie)$ Department of Plant and Soil Sciences, Oklahoma State University, Stillwater, OK 74078, USA

e-mail: liuling.yan@okstate.edu

H. Jia

Applied Plant Genomics Laboratory, Crop Genomics and Bioinformatics Centre, Nanjing Agricultural University, Nanjing, China dataset, compared with 57 cultivars and breeding lines with various genetic backgrounds. Unique sequences of the QYld.osu-1BS allele for the higher grain yield in Duster were identified, and kompetitive allele specific PCR (KASP) markers for the unique sequences were developed for breeding of novel cultivars with increased grain yield in winter wheat.

Keywords Grain yield · Genetic recombination $\cdot$ KASP marker. Wheat

\section{Introduction}

Wheat (Triticum aestivum L., $2 n=6 \times=42$, AABBDD) is one of the most important crops in the world. Winter wheat, which requires a period of low temperature to accelerate the transition from vegetative to reproductive development, occupies $75 \%$ of total wheat in worldwide (Pugsley 1971; Li et al. 2013). To maximize the grain yield of the winter wheat cultivars by conventional plant breeding and biotechnology is vital to wheat improvement of the most important economic trait (Rajaram 2005; Ray et al. 2013). Numerous quantitative trait loci (QTLs) or genomic regions have been mapped for yield traits by genetic analyses on segregated biparental populations, such as recombinant inbred lines (RILs) and doubled haploid (DH) lines, as well as association analyses on germplasm from landraces to modern varieties and breeding lines (Zhang et al. 2010; Bennett et al. 2012; Liu et al. 2012; Mir et al. 2012; Bordes et al. 2014; Cui et al. 2014; Zanke et al. 2014a, b; Gao et al. 2015; 
Lopes et al. 2015; Guo et al. 2016; Zhai et al. 2016; Assanga et al. 2017; Liu et al. 2018; Ma et al. 2018; Pradhan et al. 2019). Clustered QTLs were also found on the short arm of chromosome 1B, where pleiotropic effects on yield per plant, spikes per plant, spike length, grains per spike, thousand grain weight, and dry matter were observed (Quarrie et al. 2005; Wang et al. 2009; Sukumaran et al. 2015; Zhai et al. 2016). For grain yield as a trait, a major QTL was also located on chromosome 3AS (Baenziger et al. 2011; Rustgi et al. 2013), chromosome $5 \mathrm{~A}$ in a diverse panel of spring wheat under drought stress (Lopes et al. 2015), to the Vrn-B1 locus on chromosome 5B (Guedira et al. 2016; Sehgal et al. 2017). Vrn-B1 is a major vernalization gene that plays a crucial role, by using RIL populations derived from a cross between two soft winter wheat lines or by using CIMMYT wheat GWAM panel of 720 lines to test across diverse environments (Yan et al. 2003, 2004; Guedira et al. 2016; Sehgal et al. 2017). However, due to multiple loci following complex genetic interactions with environments, almost all of the previous yield QTLs account for only small parts of the phenotypic variation in grain yield, which has inhibited rapid utilization of the yield-related genes in wheat breeding (Quarrie et al. 2005; Bolot et al. 2009; Liu et al. 2012; Mir et al. 2012).

While the genetic effects of the genes/QTLs on grain yield are minor or genetic source for dramatic increases of grain yield in a gene pool in common wheat is lacking, alien genes from distant and closely related species of common wheat were exploited to improve grain yield (Huang et al. 2003). A genome fragment involving the short arm of rye chromosome 1R (1RS) was translocated into wheat, because the wheat-rye translocation provided resistance to insects and diseases while improved water-use efficiency and grain yield potential. The resulting 1BL.1RS translocation was used intensively in wheat breeding programs worldwide including those in the Southern Great Plains (Singh et al. 1998; Foulkes et al. 2002; Sukumaran et al. 2015). The $1 \mathrm{BL}-1 \mathrm{RS}$ translocation improved grain yield by increasing root and shoot dry weight under drought treatments, and increasing efficiencies of light conversion hence biomass (Singh et al. 1998; Foulkes et al. 2002; Ehdaie et al. 2003; Hoffmann 2008). However, the introgressed chromosome fragment carrying the desirable gene often introduces undesirable traits resulting in reduction of grain quality (Howell et al. 2014). New QTLs/genes that approach or even exceed the yield benefit of 1BL-1RS are greatly needed in the winter wheat.

The precise location of the gene for a QTL on a chromosome remains unknown in previous studies due to the lack of whole genome reference sequence, hence making it difficult to effectively utilize in wheat breeding. A draft sequence of the wheat genome was recently released by the International Wheat Genome Sequencing Consortium (IWGSC 2018), and the chromosomebased sequences provided a powerful tool for identification of physical locations of the markers developed using genotyping-by-sequencing approaches (Poland and Rife 2012; Poland et al. 2012). The identified markers linking with grain-related traits are useful to screen germplasm for favorable alleles. This study reported a major gene for grain yield and its deployment by kompetitive allele specific PCR (KASP) markers in winter wheat.

\section{Materials and methods}

Winter wheat population derived from Duster $\times$ Billings

In a previous study ( $\mathrm{Li}$ et al. 2015), a doubled haploid (DH) population was developed from two locally adapted winter wheat cultivars, "Duster" (PI 644016) and "Billings" (PI 656843), both of which are released by the Oklahoma Agricultural Experiment Station. Duster and Billings have occupied significant acreages in the Southern Great Plains due to their outstanding yielding ability (Edwards et al. 2012; Hunger et al. 2014). While Duster offers yield protection across a diverse set of environmental conditions (Edwards et al. 2012), Billings shows larger kernel size and superior yield ability in high-yielding environments (Hunger et al. 2014).

The Duster $\times$ Billings population of $260 \mathrm{DH}$ lines was tested in the field at the Stillwater Agronomy Research Station in 2014 (a drought year) and 2015 (a year with wide precipitation swings). The DH lines were arranged in a replicates-in-sets design with two replications in six sets for measuring grain in the field experiments. Each line was planted in two rows, and the single-row plots were $1 \mathrm{~m}$ long, spaced $0.5 \mathrm{~m}$ apart.

The $\mathrm{F}_{3} \# 68$ lines were derived from a cross between Duster $\times$ Billings, and those plants carrying the homozygous Duster allele and those carrying the 
homozygous Billings allele at QYld.osu-1BS, a QTL mapped in this study, were tested in fall 2017 in the same field as tested for the DH population. Each line was planted in a single $1 \mathrm{~m}$ row, spaced $0.33 \mathrm{~m}$ apart with two replicate sets in randomized design. The seeding rate was 50 seeds/row. The spikelets per spike (SNS), grains per spike (KNS), and grain weight per spike (GWS) were determined from twenty main spikes in each line.

Identification of unique sequence representing the Duster allele at QYld.osu-1BS

The Duster $\times$ Billings DH population was genotyped using genotyping-by-sequencing (GBS) approach, and a total of 2358 GBS markers were subjected to linkage mapping for the DH lines (Li et al. 2015). The GBS marker sequences were used to blast IWGSC RefSeq v1.0 (https://urgi.versailles.inra.fr/blast/?dbgroup= wheat_iwgsc_refseq_v1_chromosomes), and the resulting physical locations were ordered along each chromosome.

Wheat exome capture data for 58 accessions including Duster and Billings in the coordinated agricultural project in wheat (Wheat CAP) was used to analyze genetic diversity in diploid, tetraploid, and hexaploid wheat (T3/Wheat database, https://triticeaetoolbox. org/wheat). The exon capture assay was expected to target $90 \%$ of the genes with non-redundant low-copy sequence (He et al. 2019). The sequences in the $25.3 \mathrm{Mb}$ region covering the QYld.osu-1BS locus were used to analyze for haplotypes.

PCR markers for six genes were developed to confirm the physical location of QYld.osu-1BS or to genotype germplasm. The six genes are annotated in IWGSC RefSeq v1.0, including PLT (phospholipid-transporting ATPase) for TraesCS1B01G001800 at the positon of 1,430,072 bp, Pm3-B1 for TraesCS1B02G012000 at the positon of $5,851,440 \mathrm{bp}$, ZFP4 (zinc finger protein) for TraesCS1B02G022700 at the positon of 10,104,627 bp, NAK (serine/threonine-protein kinase-NAK) for TraesCS1B01G038800 at the positon of $18,421,447$, and $d C A P 3$ for TraesCS1B02G045400 at the positon of $25,260,567 \mathrm{bp}$. Primers specific to the genes at the QYld.osu-1BS locus were designed, and PCR products representing each of Duster and Billings alleles were sequenced. Allelic variation between Duster and Billings were determined. The information for the primers and the expected sizes of PCR products is provided in Table 1.A genomic region with an extremely low recombination rate was found in the Duster $\times$ Billings DH population, and it was thus speculated that one of the parental lines might carry the $1 \mathrm{RS} / 1 \mathrm{~B}$ translocation. The $1 \mathrm{RS} / 1 \mathrm{~B}$ translocation has been observed in different wheat lines (Koebner 1995), and PCR markers for specific repetitive sequences can effectively detect small 1RS fragments from rye genome (Koebner 1995; Saal and Wricke 1999). The $p S a D 15$ and $p S c 20 H$ are two 1 RS specific markers that are used to detect the translocated fragment from 1RS in the long arm of chromosome 1B (Liu et al. 2008; Zhai et al. 2016). In this study, three PCR markers, RIS, RYE-NOR, and SCM9 (Koebner 1995; Saal and Wricke 1999), were used to test if there is any translocated fragment from 1RS in the short arm of chromosome 1B in Duster or Billings. The information for the primers and the expected sizes of PCR products is provided in Table 1. PCRs for the above markers were performed in Quickload Master Mix (BioLabs) under the following conditions: denature at $94{ }^{\circ} \mathrm{C}$ for $5 \mathrm{~min}$, amplification for 35 cycles at $94{ }^{\circ} \mathrm{C}$ for $30 \mathrm{~s}, 55-$ $60{ }^{\circ} \mathrm{C}$ (depending on $\mathrm{Tm}$ of primers) for $30 \mathrm{~s}$, and $72{ }^{\circ} \mathrm{C}$ for $0.5-2 \mathrm{~min}$ (depending on the lengths of PCR products) per cycle, and final extension at $72{ }^{\circ} \mathrm{C}$ for $10 \mathrm{~min}$.

Development of KASP-based assays for QYld.osu-1BS

In order to speed up the selection process, three KASPbased markers for unique sequence representing the Duster allele for higher grain yield were developed in this study. KASP primers were designed following standard KASP guidelines (LGC Genomics, Hoddeson, UK), and the primer sequences are provided in Table 1. The allele-specific forward primers were designed to carry the standard FAM (5'-GAAGGTGACCAAGT TCATGCT-3') and HEX (5'-GAAGGTCGGAGTCA ACGGATT-3') tails with the targeted SNP at the 3' end, and a specific reverse primer was designed. FAM tail was added to Duster allele-specific forward primer for all three markers. The total amplicon length of expected PCR products was less than $200 \mathrm{bp}$. The

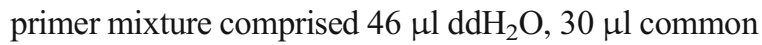
primer $(100 \mu \mathrm{M})$, and $12 \mu \mathrm{l}$ of each tailed primer $(100 \mu \mathrm{M})$. Assays were tested in 96-well formats and 
Table 1 Primers and PCR markers used in the study

\begin{tabular}{|c|c|c|c|c|}
\hline Gene/locus & Primer & Primer Sequence $\left(5^{\prime}-3^{\prime}\right)$ & $\begin{array}{l}\text { Product size (bp) } \\
\text { Duster/Billings allele }\end{array}$ & Enzyme \\
\hline \multirow[t]{2}{*}{ TraesCS1B01G001800 } & PLT-BF2 & GCGTGATGCAGCAAATAG & $1400 / 1200$ & \\
\hline & PLT-BR2 & GGTTGAGAAGGACGGCGTGATCGT & & \\
\hline \multirow[t]{2}{*}{ TraesCS1B02G012000 } & Pm3-BF2 & ACAATTTCGAACCTCATGGTGAGCTA & Null/1920 & \\
\hline & Pm3-BR3 & AGACGTGCTGCATACCTTCATAAT & & \\
\hline \multirow[t]{2}{*}{ TraesCS1B02G022700 } & ZFP4-BF4 & CTAGTTTATCAGATTAGTGTCCTACTATCCG & $500 / 400+100$ & BclI \\
\hline & ZFP4-BR4 & GAAACCTTGACATTGTAGGCGTTC & & \\
\hline \multirow[t]{2}{*}{ TraesCS1B02G037100 } & NMR-BF8 & TCAGTTCGCATTTGTGGGAGRATTTA & $410 / 400$ & \\
\hline & NMR-BR3 & AAGTAAAACTCCGGCCGTCCA & & \\
\hline \multirow[t]{2}{*}{ TraesCS1B02G038800 } & NAK-BF2 & GCCACGAGAAACTTCCGTCCG & $1600 / 750+850$ & FspI \\
\hline & NAK-BR2 & ACGACGATCTCGAGCCGTTCAC & & \\
\hline \multirow[t]{2}{*}{ TraesCS1B02G045400 } & dCAP3-F1 & GCAATCTCTTAAGGATTTCTTATGGA & $200+20 / 220$ & DpnII \\
\hline & dCAP3-R1 & CGCTTTTAGATGTTAGCTGGCAT & & \\
\hline \multirow[t]{6}{*}{ Rye 1BL.1RS } & RIS-F & TAATTTCTGCTTGCTCCATGC & 110 & \\
\hline & RIS-R & ACTGGGGTGCACTGGATTAG & & \\
\hline & RYE-NOR-F & GCATGTAGCGACTAACTCATC & $400,600,700$ & \\
\hline & RYE-NOR-R & CCCAGTTTTCCATGTCGC & & \\
\hline & SCM9-F & TGACAACCCCCTTTCCCTCGT & 200 & \\
\hline & SCM9-R & TCATCGACGCTAAGGAGGACCC & & \\
\hline \multirow[t]{3}{*}{ TraesCS1B01G001000 } & KASP-1B-12-DF & GCCTCGCCCGCGCT & & \\
\hline & KASP-1B-12-BF & GCCTCGCCCGCGCC & & \\
\hline & KASP-1B-12-CR & CGAGAAGAAGCGTCTCACGGTT & & \\
\hline \multirow[t]{3}{*}{ TraesCS1B02G022700 } & KASP-1B-17-DF & TCTTGTGAAGCTTGGGTTTCA & & \\
\hline & KASP-1B-17-BF & TCTTGTGAAGCTTGGGTTTCG & & \\
\hline & KASP-1B-17-CR & GGACCTTGTTAGGAGTGATGTATCA & & \\
\hline \multirow[t]{3}{*}{ TraesCS1B02G038800 } & KASP-1B-8-DF & CGAAAAGGAGCAAGACAAACCAT & & \\
\hline & KASP-1B-8-BF & CGAAAAGGAGCAAGACAAACCAA & & \\
\hline & KASP-1B-8-CR & GTACCTCCAGCCATCCTCTAC & & \\
\hline
\end{tabular}

set up as around $10 \mu \mathrm{l}$ reactions $(4.83 \mu \mathrm{lDNA}$ at $40 \mathrm{ng} /$ $\mu \mathrm{l}, 5 \mu \mathrm{l}$ of $1 \times$ KASP master mixture, and $0.14 \mu \mathrm{l}$ of primer mixture). PCR cycling was performed using the following protocol: hot start at $94{ }^{\circ} \mathrm{C}$ for $15 \mathrm{~min}$, followed by ten cycles of touchdown PCR $\left(94{ }^{\circ} \mathrm{C}\right.$ for $20 \mathrm{~s}$; touchdown at $61^{\circ} \mathrm{C}$ initially and decreasing by $-0.6^{\circ} \mathrm{C}$ per cycle for $60 \mathrm{~s}$ ), followed by 40 additional cycles ( $94{ }^{\circ} \mathrm{C}$ for $20 \mathrm{~s} ; 55^{\circ} \mathrm{C}$ for $60 \mathrm{~s}$ ). PCRs were performed in a real-time PCR cycler (ABI-7500) and PCR products were read in a fluorescence scanner following manufacturer's instruction (http://www.kbioscience.co. uk/reagents/KASP_manual.pdf). Testing was performed following the guideline of "Guide to running KASP genotyping on the ABI 7500 instrument" from LGC.

\section{Results}

Genetic map of QYld.osu-1BS

The average of grain yield of 12 Duster plots was $1834.3 \mathrm{~kg} / \mathrm{ha}$, whereas the average of grain yield of 12 Billings plots was $1675.7 \mathrm{~kg} / \mathrm{ha}$, which showed a significant difference in $2014(P<0.05)$. The two parental lines showed a highly significant difference in the average of grain yield of 12 plots tested in $2015,1834.3 \mathrm{~kg} /$ ha for Duster and $1643.7 \mathrm{~kg} / \mathrm{ha}$ for Billings $(P<0.001)$. The average of grain yield of the 260 Duster $\times$ Billings DH lines was $1564.91 \mathrm{~kg} / \mathrm{ha}$ ranged from 793.55 to $2427.73 \mathrm{~kg} / \mathrm{ha}$ in 2014 , whereas the average of grain yield of the same population was $1600.55 \mathrm{~kg} / \mathrm{ha}$ ranged 
from 840.63 to $2535.33 \mathrm{~kg} / \mathrm{ha}$ in 2015 . Statistical analysis showed a high correlation between grain yield scored in 2 years $(r=0.60, P<0.001)$. The results suggested that the Duster $\times$ Billings population segregated for grain yield, regardless of grain yield performed in the drought year in 2014 or the wide precipitation year in 2015 , and could be used for mapping of genes for grain yield.

A total of 2358 GBS markers was assembled into 26 linkage groups, forming genetic maps for the Duster $\times$ Billings DH population (accession number SRP051982). On the basis of whole-genome QTL scanning using interval mapping (IM) analysis, a QTL for grain yield was found in linkage group 8 (Fig. 1a). The linkage group 8, consisting of 197 GBS SNP markers, was assigned to chromosome 1B in the winter wheat. The total length of the linkage group was $120.32 \mathrm{cM}$ in genetic distance, with a marker density of $0.61 \mathrm{cM}$ per marker. The linkage group almost covered the whole chromosome $(689 \mathrm{Mb})$ from $G B S 12138$ at position of $3,569,604 \mathrm{bp}(3.6 \mathrm{Mb})$ to $G B S 575$ at $674,662,328$ bp $(674.7 \mathrm{Mb})$.

The QTL on the short arm of chromosome 1B for grain yield was referred to as QYld.osu-1BS. The logarithm of the odds (LOD) value at the peak position of QYld.osu-1BS for grain yield was 7.9 in 2014 and 13.2 in 2015, and this QTL explained $13.9 \%$ and $23.5 \%$ of the total phenotypic variation in field-based grain yield tested in 2014 and 2015, respectively. At the QYld.osu-1BS locus, Duster carried the allele for higher grain yield whereas Billings carried the allele for lower grain yield. At GBS08246 linking with the peak of QYld.osu-1BS, the average grain yield was $1681.3 \mathrm{~kg} / \mathrm{ha}$ in 2014 and $1748.5 \mathrm{~kg} / \mathrm{ha}$ in 2015 in the DH lines carrying the Duster allele but $1479.5 \mathrm{~kg} / \mathrm{ha}$ in both 2014 and 2015 in the DH lines carrying the Billings allele. The Duster allele at the QYld.osu-1BS locus increased yield by $13.6 \%$ in 2014 and $18.2 \%$ in 2015, relative to the Billings allele, indicating a significant difference in grain yield between the two alleles.

The QYld.osu-1BS is mapped to an $18.4 \mathrm{Mb}$ interval

QYld.osu-1BS is located in the distal region of chromosome arm 1BS (Fig. 1a). The peak of QYld.osu-1BS was linked with 40 clustered GBS markers, and no crossover was observed among 260 DH lines (Fig. 1a). The clustered markers cover the region from GBS12138 at the 3.6 Mb position to $G B S 9911$ at the $22.3 \mathrm{Mb}$ position. A gap was observed between GBS9911 at the $22.3 \mathrm{Mb}$ and GBS10313 at $168.6 \mathrm{Mb}$ (Fig. 1b), and 33 crossovers were found in the $146 \mathrm{Mb}$ gap between the two neighbor markers.

PCR markers for four genes confirmed the physical location of QYld.osu-1BS, including PLT at $1.4 \mathrm{Mb}, P m 3-B 1$ at $5.8 \mathrm{Mb}, Z F P 4$ at $10.1 \mathrm{Mb}$, and $N A K$ at $18.4 \mathrm{Mb}$ (Fig. 2a-d). A PCR marker, $d C A P 3$ for TraesCS1B02G045400 at the position of 25,260,567 bp, was developed (Fig. 2e). The same pair of primers were used to perform PCRs with Duster and Billings, and PCR products with expected sizes were directly sequenced and the sequence reactions worked well, indicating a single copy of the PCR products. The PCR products were distinguished between the two alleles by using restriction enzyme DpnII to do digestion. The $d C A P 3$ marker was used to narrow the QTL region from $168.6 \mathrm{Mb}$ down to $25.3 \mathrm{Mb}$ from the telomere of the short arm of chromosome $1 \mathrm{~B}$.

A large mapping population was developed from a cross between Duster and Billings to narrow down the $25.3 \mathrm{Mb}$ region of QYld.osu-1BS. A total of 4146 individual plants within $\mathrm{F}_{2: 4}$ lines were genotyped using flanking markers, and 28 recombinant events were observed, including two from $196 \mathrm{~F}_{2}$ plants, two from $1390 \mathrm{~F}_{3}$ plants, and 24 plants from $2560 \mathrm{~F}_{4}$ plants (Fig. 1c). After fine mapping of internal markers, no recombination event was observed within the region from the telomere to the marker $N A K$ at the position of $18.4 \mathrm{Mb}$ and all of the recombinant events were found to have occurred between $N A K$ at $18.4 \mathrm{Mb}$ and $d C A P 3$ at the position of $25.3 \mathrm{Mb}$. These results indicated abnormal recombination rate in the distal region of the short arm of chromosome 1B between Duster and Billings.

An $F_{3}$ population derived from the \#68 $F_{2}$ plant was tested in the field, and the $F_{2}$ plant showed the homozygous Duster allele at $d C A P 3(25.3 \mathrm{Mb})$ but heterozygous allele at $N A K(18.4 \mathrm{Mb})$ and PLT $(1.4 \mathrm{Mb})$. Those plants carrying the homozygous Duster allele and those carrying the homozygous Billings allele at QYld.osu-1BS were compared for phenotypes. As a result, the homozygous lines carrying the Duster allele at both PLT and NAK 


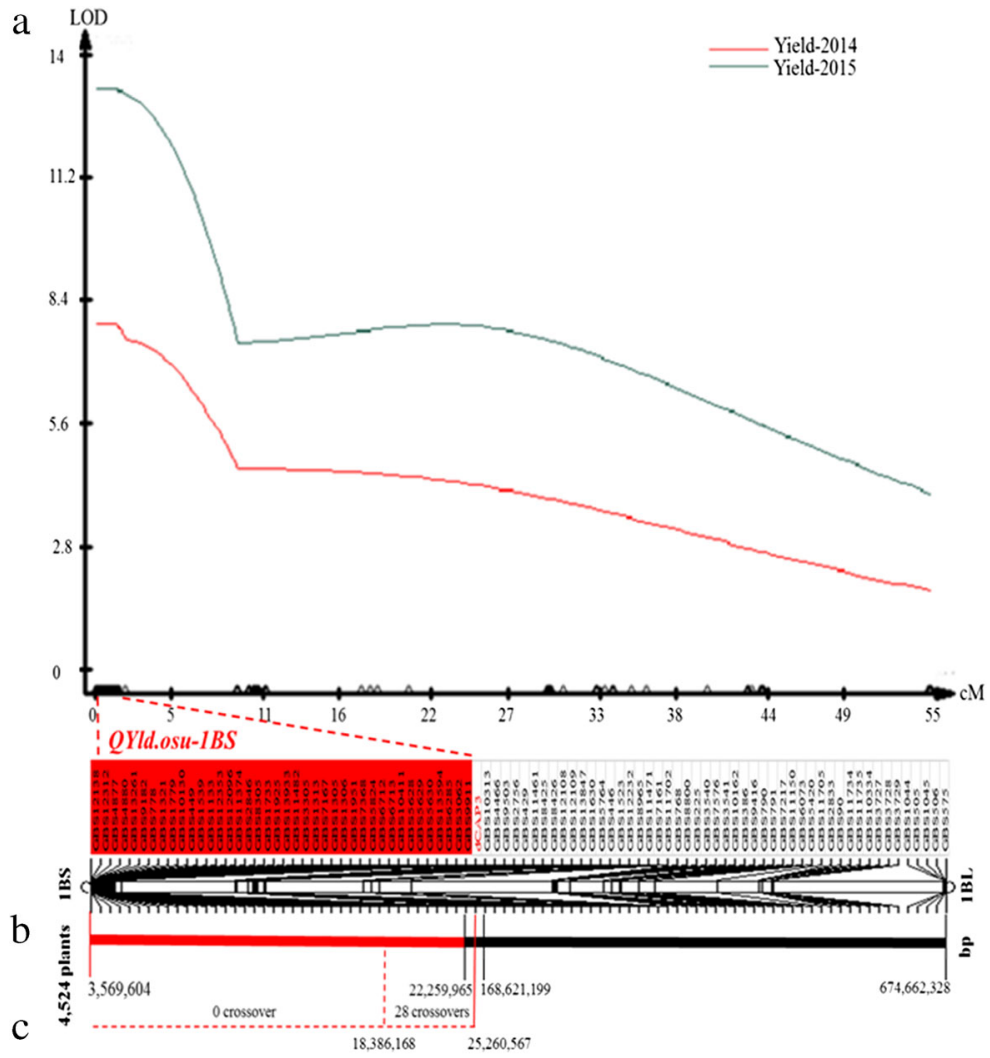

Fig. 1 Genetic mapping and physical location of QYld.osu-1BS. (a) QYld.osu- $1 B S$ is mapped to the distal region of chromosome arm 1BS. Yield-2014 and Yield-2015 indicate grain yield that was scored in the field in year 2014 and year 2015, respectively. GBS markers were used to construct the linkage group using MapMaker 3.0, with the Kosambi mapping function to estimate the map distance. WinQTLCart 2.5 (North Carolina State University, Raleigh) was used to identify QTL using composite interval mapping (CIM). The QTL was declared since the logarithm of the odds (LOD) score exceeded the threshold of 2.5 (the dotted line) that

increased $2.94 \%$ in SNS, $7.20 \%$ in KNS, and $8.87 \%$ in GWS, compared with the lines carrying the homozygous Billings allele at the two markers (Table 2).

No alien fragment was detected in Duster or Billings

The 1BL.1RS translocation contributes $9 \%$ greater yield in NE in the absence of disease bias, and the near iso-chromosome stocks developed in the late 1980 s were used intensively in wheat breeding programs in the Southern Great Plains (Moreno-Sevilla et al. 1995). In order to investigate if the 1BL.1RS was unexpectedly introduced into either Duster or Billings or their pedigrees, available markers for was determined at 300 permutations and significance level at 0.05 . Forty GBS markers that are clustered as a group and show no crossover are shaded in red. The dCAP PCR marker dCAP3 is highlighted in red. (b) The physical location of QYld.osu-1BS. QYld.osu-1BS was initially mapped to cover the telomere to $168,621,199$ bp but narrowed down to $25,260,567$ bp by dCAP3 (c) The locations of 28 crossovers in the mapping population. All 28 recombinant events were found in the region between $18.4 \mathrm{Mb}$ and $25.3 \mathrm{Mb}$

1RS translocations, including RIS, NOR, and $S C M 9$, were used to genotype the two parental lines. When the 1BL.1RS translocation was observed in Gallagher, it was not detectable in Duster or Billings using these PCR markers (Fig. 2f-h).

The exome capture assay was used to re-sequence the coding sequences of 58 wheat accessions including Duster and Billings in the Wheat CAP project. The variant calling using the GATK-based pipeline was performed, resulting in 1454 polymorphisms between Duster and Billings spanning over QYld.osu-1BS. Either Duster or Billings did not have identity to any of diploid wheat lines used in the project, suggesting neither of the two cultivars has translocation from any of the known genomes. 


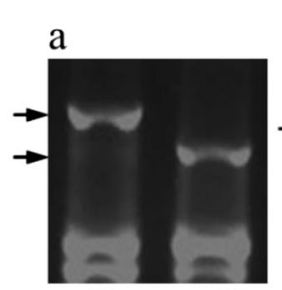

$\mathrm{D}$

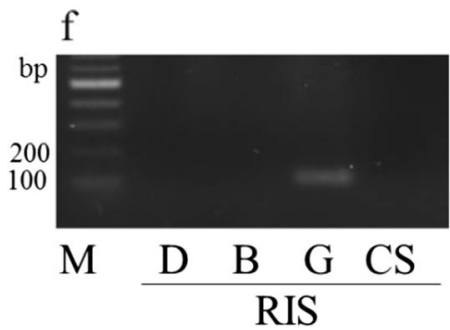

Fig. 2 PCR markers mapped at the QYld.osu- $1 B S$ locus. PCR markers were developed based on allelic variation between Duster and Billings. Arrows indicate PCR products from the Duster and Billings alleles. (a) PLT, $1400 \mathrm{bp}$ for Duster and $1200 \mathrm{bp}$ for the Billings allele; (b) Pm3B, a null allele for Duster but $1920 \mathrm{bp}$ for Billings; (c) ZFP4, 500 bp for Duster and $400 \mathrm{bp}$ and $100 \mathrm{bp}$ for Billings; (d) NAK, 1600 bp for Duster, and 850 bp and 750 bp for Billings; (e) dCAP-1B-3, $200 \mathrm{bp}$ and $20 \mathrm{bp}$ for Duster, and $220 \mathrm{bp}$

Identification of unique sequences in Duster and development of KASP markers for QYld.osu-1BS

Eighteen genes at the QYld.osu-1BS locus were selected for sequencing. Duster showed unique sequences in three genes, ZFP4, CLP for cyclophilin-like protein (TraesCS1B02G011100), and NMR for nitrogen metabolic regulator (TraesCS1B02G037100). In the NMR$B 1$ gene sequence, whereas Billings was $100 \%$ identical to $\mathrm{CS}$, Duster was unique compared with the orthologous and homoeologous genes in diploid,

Table 2 Phenotypes of yield components in $\mathrm{F}_{3}-68$ lines

\begin{tabular}{llrllllll}
\hline Genotype & Trait & \multicolumn{1}{l}{ AAA } & \multicolumn{5}{c}{ BBA } \\
\hline Phenotype & SPS & $19.05^{*}$ & \pm & 1.15 & 18.51 & \pm & 1.08 \\
& KNS & $41.20^{*}$ & \pm & 6.55 & 38.43 & \pm & 7.46 \\
& GW $(\mathrm{g})$ & $1.31^{*}$ & \pm & 0.31 & 1.20 & \pm & 0.28
\end{tabular}

$\overline{S P S \text {, spikelet number per spike; } K N S \text {, kernels per spike; } G W \text {, grain }}$ weight per spike

Numbers were averaged from 20 main spikes of three independent lines for each genotype

$A A A$, lines with Duster haplotype from PLT to NAK; $B B A$, lines with Billings genotype from PLT to NAK

$*=$ Student's $t$ test $p$ value $<0.05$

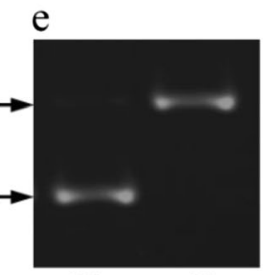

D

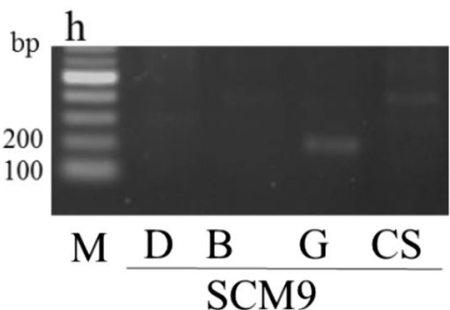

for Billings. ( $\mathrm{f}-\mathrm{h}$ ) PCR markers for rye translocated fragment in wheat, including RIS, NOR, and SCM9. Wheat lines carrying 1BL.1RS translocation yielded a $110 \mathrm{bp}$ product for RIS; $400 \mathrm{bp}, 600 \mathrm{bp}, 700 \mathrm{bp}$ for NOR-Rye, and $220 \mathrm{bp}$ for SCM9. No PCR products from those lines without translocation. M: markers; D: Duster, B: Billings, G: Gallagher as positive control line carrying the 1 RS translocation, CS: Chinese Spring as negative control

tetraploid, and hexaploid wheat (Fig. 3). A PCR marker for the unique sequence at $N M R$ in Duster was used to screen up to 135 accessions, only five accessions showed the same allele as Duster (Supplementary Table S1).

Duster was a unique haplotype in the targeted QYld.osu-1BS region, compared with the other 57 cultivars and breeding lines with various genetic backgrounds that were used for exome capture. Among 6820 SNPs in the 25.3 Mb QYld.osu-1BS region, Duster had 1102 bp of unique SNPs compared with Billings and Chinese Spring. In comparison, Billings had $432 \mathrm{bp}$ of unique SNPs compared with Duster and Chinese Spring. The 1064 bp (96.5\%) out of the 1102 SNPs in Duster occurred in the region from the telomere to the marker $N A K$ at the position of $18.4 \mathrm{Mb}$ (Fig. 4a). In addition, among 58 accessions, Duster has 928 SNPs representing minor allele, and $718(77 \%)$ out of the 928 SNPs in Duster were found to be located at the genomic region between 5 and $12.5 \mathrm{Mb}$ (Fig. 4b).

The unique sequences in Duster were converted into high-throughput KASP assays. KASP-12 was designed for the SNP at 1,253,260 bp (Fig. 5a), KASP-17 was designed for the SNP at 10,104,175 bp (Fig. 5b), and KASP-8 was designed for the SNP at $18,386,168 \mathrm{bp}$ (Fig. 5c). The unique markers representing the Duster 


\begin{tabular}{l}
\hline \\
NMR-B1-Duster \\
NMR-B1-Billings \\
NMR-B1-CS \\
NMR-A1-CS \\
NMR-D1-CS \\
NMR-speltoides \\
NMR-sharonensis \\
NMR-uratu \\
NMR-monococcum \\
NMR-tauschii \\
NMR-durum1 \\
NMR-durum2
\end{tabular}

NMR-B1-Duster

NMR-B1-Billings

NMR-B1-CS

NMR-A1-CS

NMR-D1-CS

NMR-speltoides

NMR-sharonensis

NMR-uratu

NMR-monococcum

NMR-tauschii

NMR-durum 1

NMR-durum2

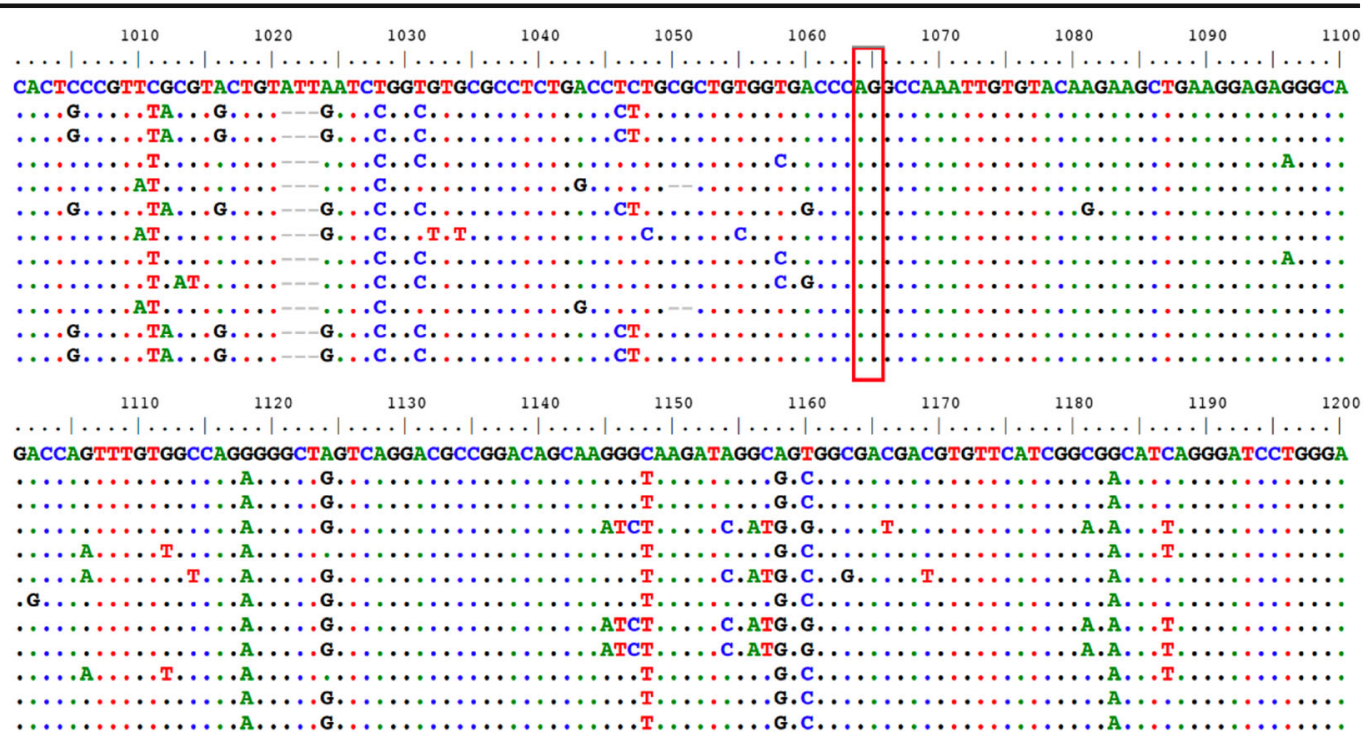

Fig. 3 Multiple sequence alignment of the $N M R-B 1$ genes. Unique sequences were found throughout $2150 \mathrm{bp}$ of the complete $N M R-B 1$ gene including conserved exon regions in Duster. The partial sequences including exon 2 of the $N M R-B 1$ genes are

allele spanning QYld.osu-1BS were used to ensure that the whole genomic region of the Duster allele at QYld.osu-1BS is introduced into novel lines/varieties.

\section{Discussion}

In this study, QYld.osu-1BS, as a major QTL for grain yield, was discovered in Duster, which accounted for $18.7 \%$ of the phenotypic variation in grain yield of the entire population tested for 2 years. The Duster allele at QYld.osu-1BS increased $15.9 \%$ in field-based grain yield, compared with the Billings allele. QTLs for yield and yield components have been reported to account for only small parts of the phenotypic variation, because the QTLs may be subject to large genotype-environment interactions (Lopes et al. 2015; Kirigwi et al. 2007). The major gene at QYld.osu-1BS in Duster is one of the largest QTL influencing grain yield identified to date.

As the present study advanced, a draft sequence of the wheat genome was recently released in the International Wheat Genome Sequencing Consortium (IWGSC 2018). The chromosome-based sequences provided a powerful tool for identification of chromosomal locations of the GBS markers. QYld.osu-1BS was located in a genomic region where recombination rate was extremely low. A hypothesis accounting for the abnormal aligned to indicate difference between Duster and other sources available in the IWGSC RefSeq v1.0 database. The AG splicing site at the 3 'end of intron 1 of the NMR-B1 genes is indicated with a red rectangle

recombination rate was that the $1 \mathrm{RS}$ translocation used extensively in the breeding programs in the region was unexpectedly introduced into either Duster or Billings. The 1RS genomic fragment is mostly utilized in spring wheat cultivars, but it has also been introduced into "Gallagher," a new winter wheat cultivar that was released in 2013 (PI 667569, PVP201300134). However, the results from PCR markers and exome capture sequences indicated that the low recombination at QYld.osu-1BS was not caused by $1 \mathrm{BL}-1 \mathrm{RS}$ or any alien fragment from accessions of diploid wheat species tested in the exome capture data.

Based on the phenotypes and genotypes of the $\mathrm{F}_{3}-68$ population, the gene for QYld.osu-1BS for grain yield could be located in the region from the telomere to the marker NAK at the position of $18.4 \mathrm{Mb}$. However, it should be cautious that the result was from the only one recombinant line. A final conclusion should be drawn after more lines that have crossovers between NAK at $18.4 \mathrm{Mb}$ and dCAP3 at $25.3 \mathrm{Mb}$ are tested for the phenotypes.

Positional cloning of the gene in the genomic region with low recombination rate is a difficult task, though the draft of genomic sequences in wheat has been available. If the low recombination region was resulted from the presence of an alien genomic fragment in Duster, probably in the region between the telomere to $18.4 \mathrm{Mb}$, the problem could be solved by introduction of $P h 1$ 


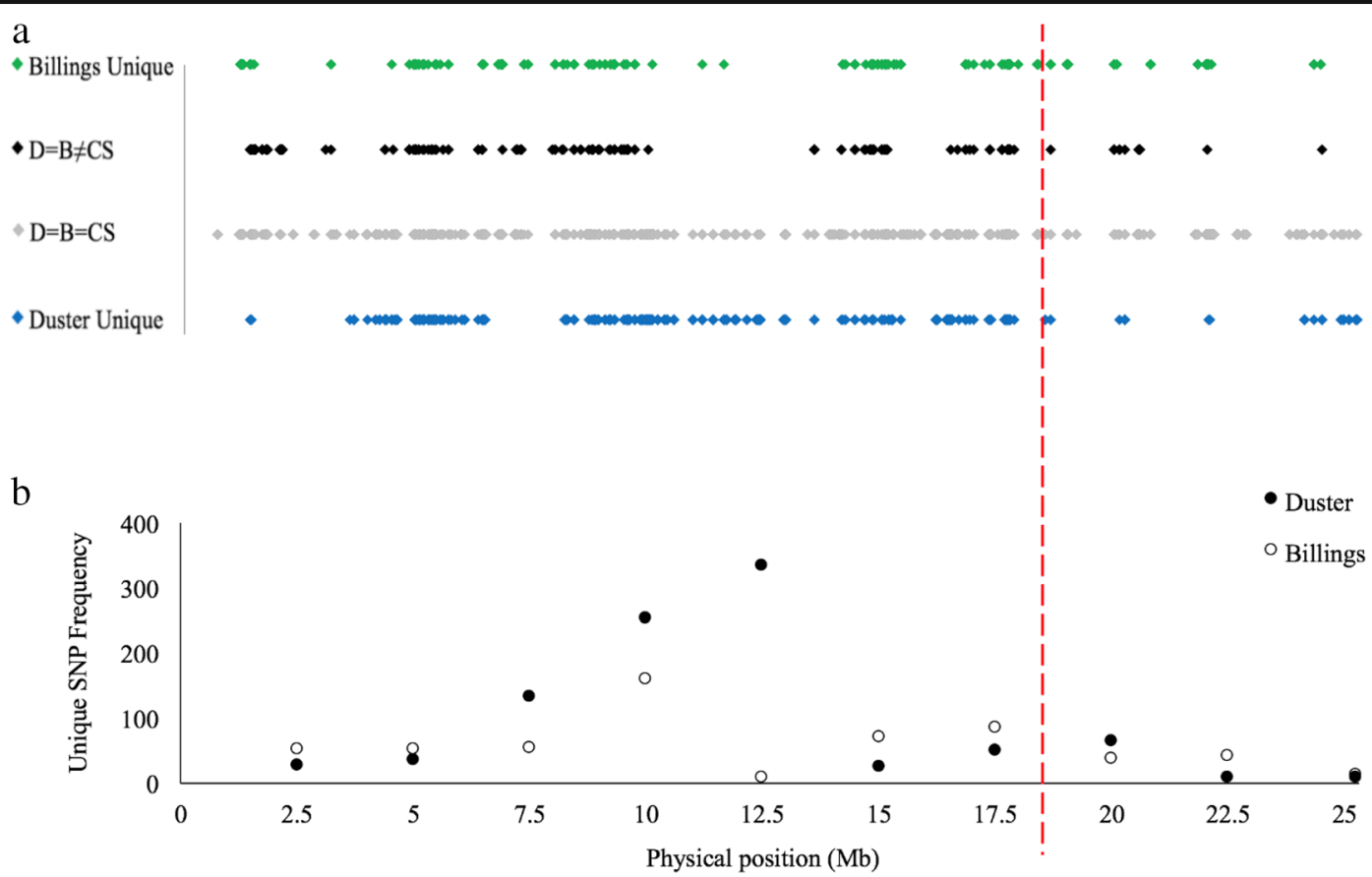

Fig. 4 Distribution of unique sequences in Duster along the QYld.osu-1BS. a Distribution of exome captured SNPs across the $25.3 \mathrm{Mb}$ region in different genotypes. Allelic relationship among Duster (D), Billings (B), and Chinese Spring (CS) are color coded. b Unique SNPs are counted when the allele is minor allele among

gene (Dover and Riley 1972). Increased recombination events could be used to clone the gene responsible for QYld.osu-1BS, but it would take several years to introduce the $P h 1$ gene and purify genetic backgrounds brought from genetic materials carrying the $P h 1$ gene. Another approach for cloning of the gene is to create mutants of the genes in the targeted region by using EMS and validate the functions of the genes (Krasileva et al. 2017), but it could be too difficult to validate up to 360 genes with high confidence in the QYld.osu-1BS locus. Before the gene is cloned, the unique sequences the 58 accessions. Frequencies of unique Duster or Billings SNPs are shown every $2.5 \mathrm{Mb}$ from the telomere to $25.3 \mathrm{Mb}$ on chromosome 1BS. Red dot line indicates the position of NAK at $18.4 \mathrm{Mb}$

identified in the QYld.osu-1BS region can be used as molecular markers for accelerating deployment of the gene for QYld.osu-1BS in conventional wheat breeding programs.

While the genetic mechanisms underlying the high grain yields in the two winter wheat cultivars remain a mystery, there is still substantial potential in exploitation of wheat yield genes per se, in cultivars currently deployed in wheat production. The major QYld.osu-1BS for grain yield was found in the locally adapted cultivar, allowing it to readily breed new wheat varieties. The
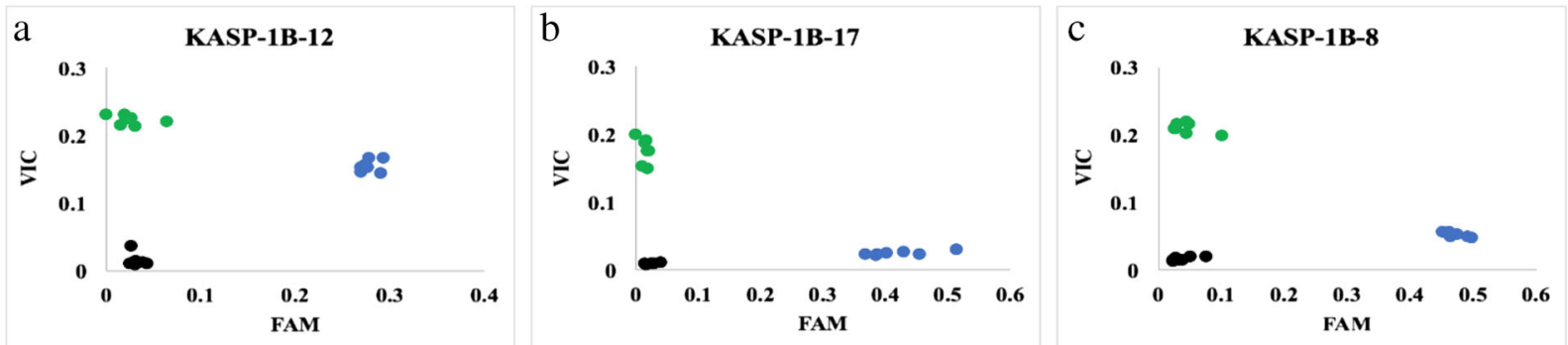

Fig. 5 Three KASP markers developed for SNPs unique in Duster to cover QYld.osu-1BS. a KASP-12 at 1,253,260 bp. b KASP17 at $10,104,175$ bp. c KASP-12 at 18,386,168 bp. KASP-FAM showing the Duster allele is indicated in blue color, KASP-HEX showing the Billings allele is indicated in green color, and NTC (non-template control) used for controls is indicated in black dots 
QYld.osu-1B gene has been introduced into numerous lines by marker-assisted backcrossing and to deliver those lines to the hub for evaluation. Duster, has the QYld.osu- $1 B$ gene, and its offspring or grand-offspring have been introgressed in the pedigrees of $25 \%$ of the elite germplasm resident to the wheat improvement program. The research results from this study can be used to accelerate deployment of a beneficial allele for a major gene increasing grain yield, leading to more productive winter wheat varieties in diverse environments worldwide.

Author contributions C-C Kan, H Jia, C Powers, and L Yan performed the experiments and the analyzed data. B Carver developed and phenotyped the population. B Carver and L Yan designed the experiments. C-C Kan and L Yan wrote the manuscript. All authors read and approved the manuscript.

Funding information This project was supported by the Agriculture and Food Research Initiative Competitive Grants (201767007-25932) from the USDA National Institute of Food and Agriculture (NIFA), and grants from the Oklahoma Center for Advanced Science and Technology (OCAST, AR17-020-03). This study was also supported by the Oklahoma Wheat Research Foundation and the Oklahoma Agricultural Experiment Station.

\section{Compliance with ethical standards}

Conflict of interest The authors declare that they have no competing interests.

Open Access This article is licensed under a Creative Commons Attribution 4.0 International License, which permits use, sharing, adaptation, distribution and reproduction in any medium or format, as long as you give appropriate credit to the original author(s) and the source, provide a link to the Creative Commons licence, and indicate if changes were made. The images or other third party material in this article are included in the article's Creative Commons licence, unless indicated otherwise in a credit line to the material. If material is not included in the article's Creative Commons licence and your intended use is not permitted by statutory regulation or exceeds the permitted use, you will need to obtain permission directly from the copyright holder. To view a copy of this licence, visit http://creativecommons.org/licenses/by/4.0/.

\section{References}

Assanga SO, Fuentealba M, Zhang G, Tan C, Dhakal S, Rudd JC, Ibrahim AMH, Xue Q, Haley S, Chen J, Chao S, Baker J, Jessup K, Liu S (2017) Mapping of quantitative trait loci for grain yield and its components in a US popular winter wheat TAM 111 using 90K SNPs. PLoS One 12:e189669
Baenziger PS, Dweikat I, Gill KS, Eskridge K, Berke T, Shah M, Campbell BT, Ali ML, Mengistu N, Mahmood A, Auvuchanon A, Yen Y, Rustgi N, Moreno-Sevilla B, Mujeeb-Kazi A, Morris MR (2011) Understanding grain yield: it is a journey, not a destination. Czech J Genet Plant Breed 47:S77-S84

Bennett D, Izanloo A, Reynolds M, Kuchel H, Langridge P, Schnurbusch T (2012) Genetic dissection of grain yield and physical grain quality in bread wheat (Triticum aestivum L.) under water-limited environments. Theor Appl Genet 125: 255-271

Bolot S, Abrouk M, Masood-Quraishi U, Stein N, Messing J, Feuillet C, Salse J (2009) The "inner circle" of the cereal genomes. Curr Opin Plant Biol 12:119-125

Bordes J, Goudemand E, Duchalais L, Chevarin L, Oury FX, Heumez E, Lapierre A, Perretant MR, Rolland B, Beghin D, Laurent V, Gouis JL, Storlie E, Robert O, Charmet G (2014) Genome-wide association mapping of three important traits using bread wheat elite breeding populations. Mol Breed 33:755-768

Cui F, Zhao CH, Ding AM, Li J, Wang L, Li XF, Bao YG, Li JM, Wang HG (2014) Construction of an integrative linkage map and QTL mapping of grain yield-related traits using three related wheat RIL populations. Theor Appl Genet 127:659-675

Dover AG, Riley R (1972) Prevention of pairing of homoeologous meiotic chromosomes of wheat by an activity of supernumerary chromosomes of Aegilops. Nature 240:159-161

Edwards JT, Hunger RM, Smith EL, Horn GW, Chen MS, Yan L (2012) 'Duster' wheat: a durable, dual-purpose cultivar adapted to the Southern Great Plains of the USA. J Plant Regist 6:1-12

Ehdaie B, Whitkus RW, Waines JG (2003) Root biomass, wateruse efficiency, and performance of wheat-rye translocations of chromosomes 1 and 2 in spring bread wheat 'Pavon'. Crop Sci 43:710-717

Foulkes MJ, Scott RK, Sylvester-Bradley R (2002) The ability of wheat cultivars to withstand drought in UK conditions: formation of grain yield. J Agric Sci 138:153-169

Gao F, Wen W, Liu J, Rasheed A, Yin G, Xia X, Wu X, He Z (2015) Genome-wide linkage mapping of QTL for yield components, plant height and yield-related physiological traits in the Chinese wheat cross Zhou 8425B/Chinese spring. Front Plant Sci 6:1099

Guedira M, Xiong M, Hao YF, Johnson J, Harrison S, Marshall D, Brown-Guedira G (2016) Heading date QTL in winter wheat (Triticum aestivum L.) coincide with major developmental genes VERNALIZATION1 and PHOTOPERIOD1. PLOS One 11:e0154242

Guo Z, Slafer GA, Schnurbusch T (2016) Genotypic variation in spike fertility traits and ovary size as determinants of floret and grain survival rate in wheat. J Exp Bot 67:4221-4230

He F, Pasam R, Shi F, Kant S, Keeble-Gagnere G, Kay P, Forrest F, Fritz A, Hucl P, Wiebe K, Knox R, Cuthbert R, Pozniak C, Akhunova A, Morrell P, Davies J, Webb S, Spangenberg S, Hayes B, Daetwyler H, Tibbits J, Hayden M, Akhunov E (2019) Exome sequencing highlights the role of wild-relative introgression in shaping the adaptive landscape of the wheat genome. Nat Genet 51:896-904 
Hoffmann B (2008) Alteration of drought tolerance of winter wheat caused by translocation of rye chromosome segment 1RS. Cereal Res Commun 36:269-278

Howell T, Hale I, Jankuloski L, Bonafede M, Gilbert M, Dubcovsky J (2014) Mapping a region within the 1RS.1BL translocation in common wheat affecting grain yield and canopy water status. Theor Appl Genet 127:2695-2709

Huang X, Coster H, Ganal MW, Röder MS (2003) Advanced backcross QTL analysis for the identification of quantitative trait loci alleles from wild relatives of wheat (Triticum aestivum L.). Theor Appl Genet 106:1379-1389

Hunger RM, Edwards JT, Bowden RL, Yan L, Rayas-Duarte P, Bai G, Horn GW, Kolmer JA, Giles KL, Chen MS, Jin Y (2014) 'Billings' wheat combines early maturity, disease resistance, and desirable grain quality for the Southern Great Plains of the USA. J Plant Regist 8:22-31

IWGSC (2018) Shifting the limits in wheat research and breeding using a fully annotated reference genome. Science 361: eaar7191

Kirigwi FM, Van Ginkel M, Brown-Guedira G, Gill BS, Paulsen GM, Fritz AK (2007) Markers associated with a QTL for grain yield in wheat under drought. Mol Breed 20:401-413

Koebner RMD (1995) Generation of PCR-based markers for the detection of rye chromatin in a wheat background. Theor Appl Genet 90:740-745

Krasileva KV, Vasquez-Gross HA, Howell T, Bailey P, Paraiso F, Clissold L, Simmonds J, Ramirez-Gonzalez RH, Wang XD, Borrill P, Fosker C, Ayling S, Phillips AL, Uauy C, Dubcovsky J (2017) Uncovering hidden variation in polyploid wheat. Proc Natl Acad Sci U S A 114:E913-E921

Li G, Yu M, Fang T, Cao S, Carver BF, Yan L (2013) Vernalization requirement duration in winter wheat is controlled by TaVRN-A1 at the protein level. Plant J 76:742-753

Li G, Wang Y, Chen M, Edae E, Poland J, Akhunov E, Chao S, Bai G, Carver BF, Yan L (2015) Precisely mapping a major gene conferring resistance to Hessian fly in bread wheat using genotyping-by-sequencing. BMC Genomics 16:108

Liu C, Yang Z, Li G, Zeng Z, Zhang Y, Zhou J, Liu Z, Ren Z (2008) Isolation of a new repetitive DNA sequence from Secale africanum enables targeting of Secale chromatin in wheat background. Euphytica 159:249-258

Liu Y, He Z, Appels R, Xia X (2012) Functional markers in wheat: current status and future prospects. Theor Appl Genet 125:1-10

Liu K, Sun X, Ning T, Duan X, Wang Q, Liu T, An Y, Guan X, Tian J, Chen J (2018) Genetic dissection of wheat panicle traits using linkage analysis and a genome-wide association study. Theor Appl Genet 131:1073-1090

Lopes M, Dreisigacker S, Peña R, Sukumaran S, Reynolds M (2015) Genetic characterization of the wheat association mapping initiative (WAMI) panel for dissection of complex traits in spring wheat. Theor Appl Genet 128:453464

Ma F, Xu Y, Ma Z, Li L, An D (2018) Genome-wide association and validation of key loci for yield-related traits in wheat founder parent Xiaoyan 6. Mol Breed 38:91

Mir RR, Kumar N, Jaiswal V, Girdharwal N, Prasad M, Balyan HS, Gupta PK (2012) Genetic dissection of grain weight in bread wheat through quantitative trait locus interval and association mapping. Mol Breed 29:963-972
Moreno-Sevilla B, Baenziger PS, Peterson CJ, Graybosch RA, McVey DV (1995) The 1BL/1RS translocation: agronomic performance of F3-derived lines from a winter wheat cross. Crop Sci 35:1051-1055

Poland JA, Rife TW (2012) Genotyping-by-sequencing for plant breeding and genetics. Plant Genome 5:92-102

Poland JA, Brown PJ, Sorrells ME, Jannink JL (2012) Development of high-density genetic maps for barley and wheat using a novel two-enzyme genotyping-by-sequencing approach. PLoS One 7:e32253

Pradhan S, Babar MA, Robbins K, Bai G, Mason RE, Khan J, Shahi D, Avci M, Guo J, Maksud Hossain M, Bhatta M, Mergoum M, Asseng S, Amand PS, Gezan S, Baik B-K, Blount A, Bernardo A (2019) Understanding the genetic basis of spike fertility to improve grain number, harvest index, and grain yield in wheat under high temperature stress environments. Front Plant Sci 10:1481

Pugsley AT (1971) A genetic analysis of the spring-winter habit of growth in wheat. Aust J Agric Res 22:21-31

Quarrie SA, Steed A, Calestani C, Semikhodskii A, Lebreton C, Chinoy C, Steele N, Pljevljakusic D, Waterman E, Weyen J, Schondelmaier J, Habash DZ, Farmer P, Saker L, Clarkson DT, Abugalieva A, Yessimbekova M, Turuspekov Y, Abugalieva S, Tuberosa R, Sanguineti M-C, Hollington PA, Aragués R, Royo A, Dodig D (2005) A high-density genetic map of hexaploid wheat (Triticum aestivum L.) from the cross Chinese Spring $\times$ SQ1 and its use to compare QTLs for grain yield across a range of environments. Theor Appl Genet 110:865-880

Rajaram S (2005) Role of conventional plant breeding and biotechnology in future wheat production. Turk J Agric For 29: 105-111

Ray DK, Mueller ND, West PC, Foley JA (2013) Yield trends are insufficient to double global crop production by 2050. PLoS One 8:e66428

Rustgi S, Shafqat MN, Kumar N, Baenziger PS, Ali ML, Dweikat I, Campbell BT, Gill KS (2013) Genetic dissection of yield and its component traits using high-density composite map of wheat chromosome 3A: bridging gaps between QTLs and underlying genes. PLoS One 8:e70526

Saal B, Wricke G (1999) Development of simple sequence repeat markers in rye (Secale cereal L.). Genome 42:964-972

Sehgal D, Autrique E, Singh R, Ellis M, Singh S, Dreisigacker S (2017) Identification of genomic regions for grain yield and yield stability and their epistatic interactions. Sci Rep 7: 41578

Singh RP, Huerta-Espino J, Rajaram S, Crossa J (1998) Agronomic effects from chromosome translocations 7DL.7Ag and 1BL.1RS in spring wheat. Crop Sci 38: 27-33

Sukumaran S, Dreisigacker S, Lopes M, Chavez P, Reynolds MP (2015) Genome-wide association study for grain yield and related traits in an elite spring wheat population grown in temperate irrigated environments. Theor Appl Genet 128: 353-363

Wang R, Hai L, Zhang X, You G, Yan C, Xiao S (2009) QTL mapping for grain filling rate and yield-related traits in RILs of the Chinese winter wheat population Heshangmai $\times$ Yu8679. Theor Appl Genet 118:313-325

Yan L, Loukoianov A, Tranquilli G, Helguera M, Fahima T, Dubcovsky J (2003) Positional cloning of wheat 
vernalization gene VRN1. Proc Natl Acad Sci U S A 100:6263-6268

Yan L, Helguera M, Kato K, Fukuyama S, Sherman J, Dubcovsky $\mathrm{J}$ (2004) Allelic variation at the VRN-1 promoter region in polyploid wheat. Theor Appl Genet 109:1677-1686

Zanke C, Ling J, Plieske J, Kollers S, Ebmeyer E, Korzun V, Argillier O, Stiewe G, Hinze M, Beier S, Ganal MW, Roder MS (2014a) Genetic architecture of main effect QTL for heading date in European winter wheat. Front Plant Sci 5:217

Zanke CD, Ling J, Plieske J, Kollers S, Ebmeyer E, Korzun V, Argillier O, Stiewe G, Hinze M, Neumann K, Ganal MW, Röder MS (2014b) Whole genome association mapping of plant height in winter wheat (Triticum aestivum L.). PLoS One 9:e113287
Zhai H, Feng Z, Li J, Liu X, Xiao S, Ni Z, Sun Q (2016) QTL analysis of spike morphological traits and plant height in winter wheat (Triticum aestivum L.) using a high-density SNP and SSR-based linkage map. Front Plant Sci 7:1617

Zhang LY, Liu DC, Guo XL, Yang WL, Sun JZ, Wang DW, Zhang A (2010) Genomic distribution of quantitative trait loci for yield and yield-related traits in common wheat. J Integr Plant Biol 52:996-1007

Publisher's note Springer Nature remains neutral with regard to jurisdictional claims in published maps and institutional affiliations. 